\title{
Touching In Biological Systems: A 3D Force Microscope
}

${ }^{\mathbf{1 , 2}}$ Richard Superfine*, ${ }^{\mathbf{2}}$ G. Bishop, ${ }^{\mathbf{3}} \mathrm{J}$. Cummings, ${ }^{\mathbf{3}} \mathrm{J}$. Fisher, ${ }^{2} \mathrm{~K}$. Keller, ${ }^{4} \mathrm{G}$. Matthews, ${ }^{\mathbf{1} D}$. Sill, ${ }^{1,2}$ R. M. Taylor II, ${ }^{2}$ L. Vicci, ${ }^{2} \mathrm{C}$. Weigle, ${ }^{2} \mathrm{G}$. Welch, ${ }^{2} \mathrm{~B}$. Wilde.

${ }^{1}$ Department of Physics and Astronomy and Curriculum in Applied and Materials Sciences*, ${ }^{2}$ Computer Science Department, ${ }^{3}$ Biomedical Engineering, ${ }^{4}$ Cystic Fibrosis Center, University of North Carolina-Chapel Hill, Chapel Hill, NC 27599, USA

We are creating a microscopy system that allows a scientist to be an interactive actor in biological systems. Scanning probe microscopy (SPM) is being applied in a wide range of problems in biology, including the imaging of cell membranes and single molecules, and the measurement of the forces that bind ligands and maintain molecular conformations. Over the past decade we have developed user interfaces for SPM that allow the scientist to be an actor in the experiment. By moving a pen in held in hand, the user can control the motion of the probe tip on the surface, and through the force display capabilities of the pen, feel the surface and the biological sample. Applications of this interface include viruses, fibrin and nanotubes. However, performing force measurements inside cells and other biological systems remains a difficult challenge. The SPM tip, due to its overall dimensions, will strongly perturb a biological system such as a live cell or the extracellular matrix. The trick to solving this dilemma is to free the tip of the SPM probe from the cantilever.

We have developed a 3D force microscope by manipulating a freely suspended bead in liquid. By holding a pen in hand, the user can control the forces applied to and the resulting motion of a bead suspended in a biological system. The 3D force microscope uses magnetic fields to apply forces to ferromagnetic and paramagnetic spheres, and forward light scattering to track the particle. We have designed a tetrahedral electromagnetic pole geometry using finite element analysis to model the fields and forces. With pole spacing of 350 microns, we are able to generate over $100 \mathrm{pN}$ of force with air-cooled coils. A tracking algorithm allows the particle to be tracked within the 100 micron $\times 100$ micron $\times 15$ micron range of the sample stage while the forward light scattering signal allows position measurements with high spatial resolution $(<10 \mathrm{~nm})$ and bandwidth exceeding $10 \mathrm{kHz}$. The microscope has been interfaced to a haptic (force feedback) interface that allows the positioning of the particle using a hand held pen. Motion of the pen in 3D controls the coil currents, and hence the applied force, and the force is displayed to the user hand through motors that drive the pen. We present results from our initial applications in the viscoelasticity of mucus and cell studies.

Taylor II, R. M. and R. Superfine, Advanced Interfaces to Scanning Probe Microscopes. Handbook of Nanostructured Materials and Nanotechnology. H. S. Nalwa. New York, Academic Press. 2: 271-308. (1999). 


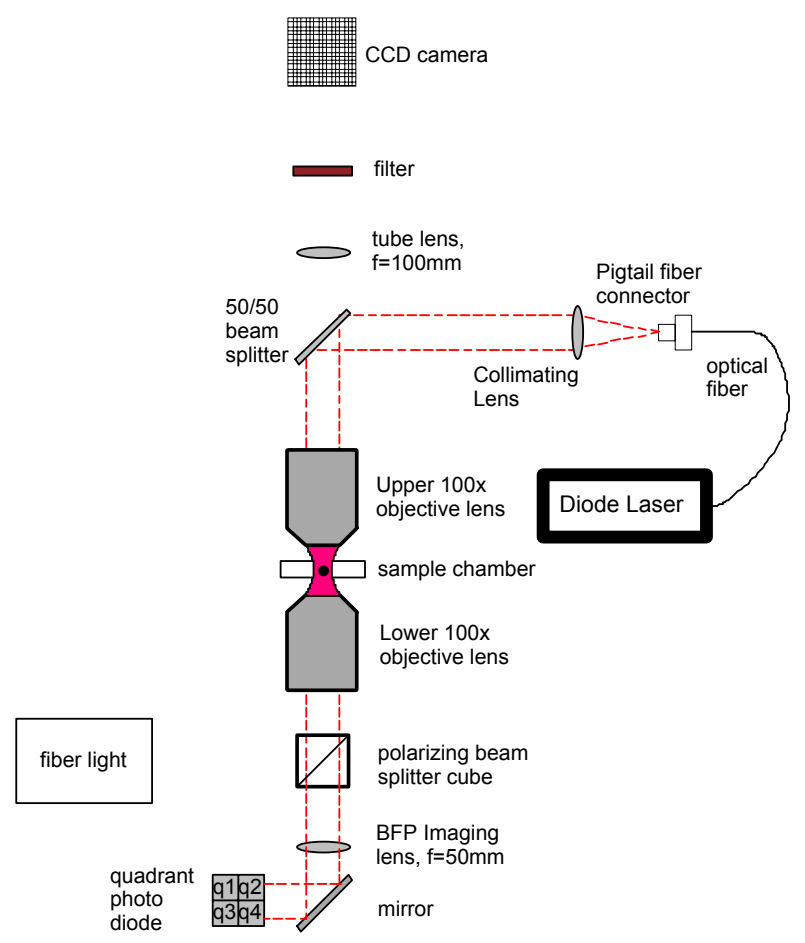

Fig. 1. Schematic of Optical tracking system for the 3DFM. This initial implementation uses $0.7 \mathrm{NA}$ objectives (Mitutoyo) to allow room for the tetrahedral pole geometry.

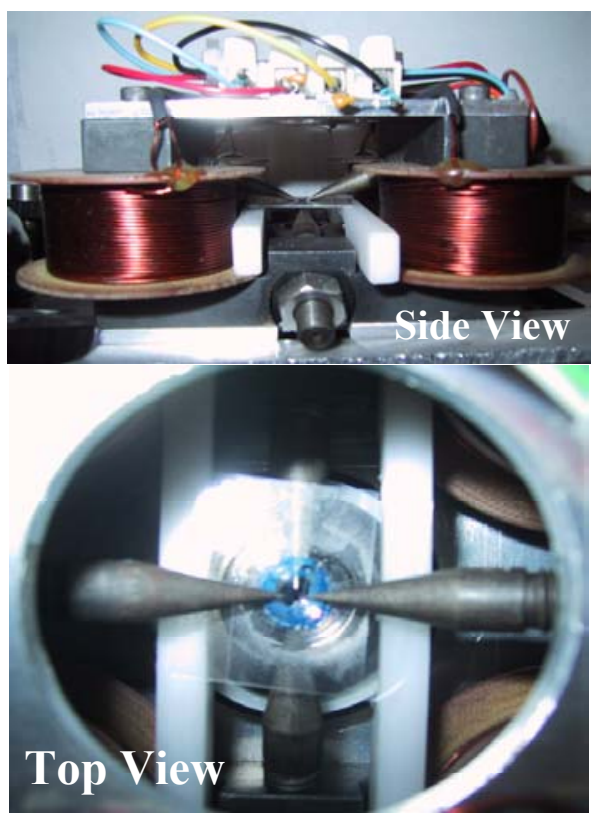

Fig. 2. Side and Top views of the tetrahedral magnetic pole geometry. Two poles project from the top, and two poles, rotated 90 degrees from the top, project from the bottom. The sample slides between the poles.
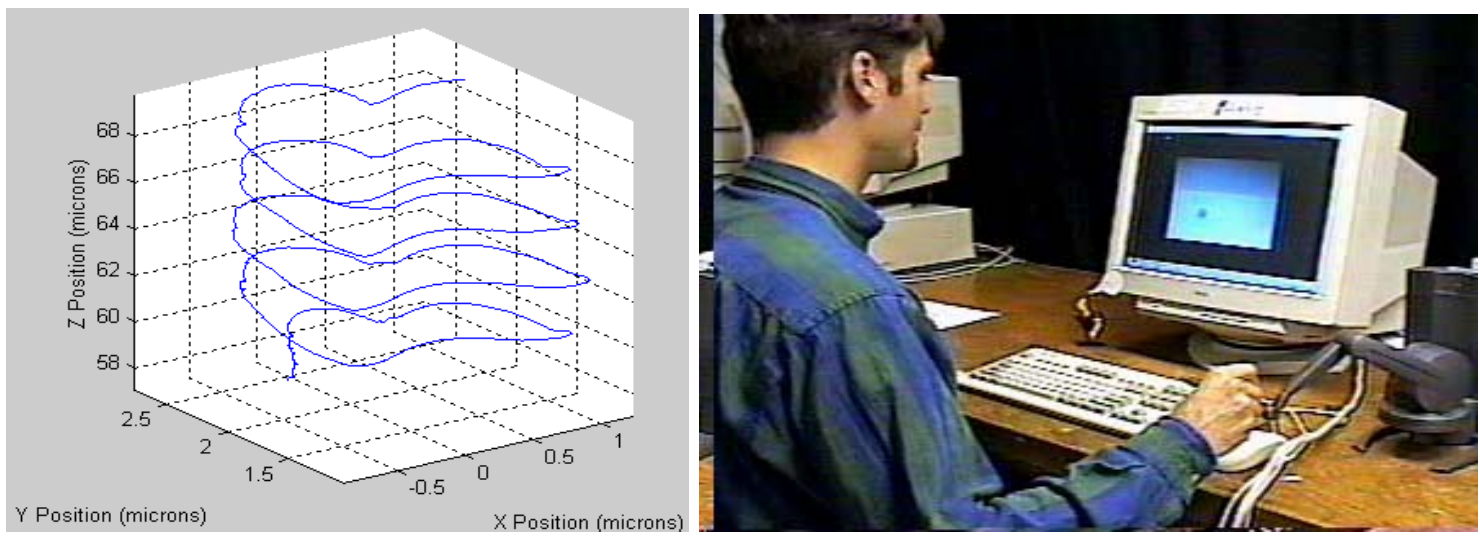

Fig. 3. J. Cummings using force feedback pen to guide magnetic bead through liquid sample.
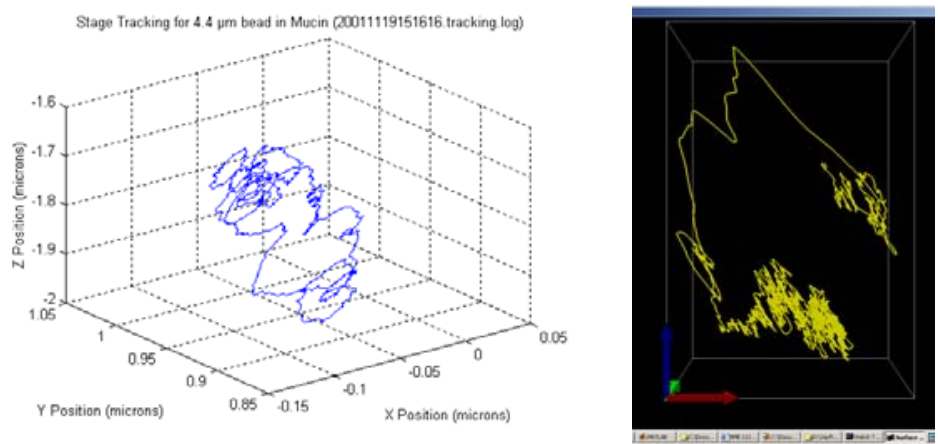

Fig. 4. Freely diffusing bead in mucus tracked in $3 \mathrm{~d}$ (far left). Bead tracked in agarose gel show signs of slipping between "cages" that confine the bead for long times. 